\title{
BMJ Open Exploring levels and correlates of health literacy in Arabic and Vietnamese immigrant patients with cancer and their English-speaking counterparts in Australia: a cross-sectional study protocol
}

Martha Gerges, ${ }^{1,2}$ Allan Ben Smith, ${ }^{1,2}$ Ivana Durcinoska, ${ }^{1}$ Henry Yan, ${ }^{1,2}$ Afaf Girgis ${ }^{1,2}$

To cite: Gerges M, Smith AB, Durcinoska I, et al. Exploring levels and correlates of health literacy in Arabic and Vietnamese immigrant patients with cancer and their Englishspeaking counterparts in Australia: a cross-sectional study protocol. BMJ Open 2018;8:e21666. doi:10.1136/ bmjopen-2018-021666

- Prepublication history for this paper is available online To view these files, please visit the journal online (http://dx.doi org/10.1136/bmjopen-2018021666).

Received 11 January 2018 Revised 21 June 2018 Accepted 27 June 2018

\section{Check for updates}

(c) Author(s) (or their employer(s)) 2018. Re-use permitted under CC BY-NC. No commercial re-use. See rights and permissions. Published by BMJ.

${ }^{1}$ Psycho-Oncology Research Group, Centre for Oncology Education and Research Translation (CONCERT) Ingham Institute for Applied Medical Research, Liverpool, New South Wales, Australia

${ }^{2}$ South Western Sydney Clinical School, The University of New South Wales, Liverpool, New South Wales, Australia

Correspondence to Dr. Allan Ben Smith; ben.smith@unsw.edu.au

\section{ABSTRACT}

Introduction For immigrants diagnosed with cancer, the stress of a cancer diagnosis and treatment can be amplified by unfamiliarity with the health system, lack of culturally and linguistically appropriate information, and inability to communicate efficiently and accurately with the treating team. Lower levels of health literacy may be one factor underlying poorer outcomes among immigrant patients with cancer, but there have been few studies exploring this issue to date. This study aims to investigate the levels and correlates of health literacy in two immigrant populations affected by cancer and their English-speaking counterparts.

Methods and analysis Levels and correlates of health and eHealth literacy will be evaluated using a crosssectional self-report questionnaire. Eligible, English, Arabic and Vietnamese patients with cancer and survivors ( $\mathrm{n}=50$ of each language group) will be invited to complete a questionnaire in their preferred language containing the Health Literacy Questionnaire, the eHealth Literacy Scale and study-specific questions assessing potential correlates of poor health literacy, including gender, age, education level, acculturation into Australian society and number of chronic illnesses. Multivariable logistic regression will be used to identify potential approaches to support effective communication with healthcare providers and preferred methods for assessing patient-reported outcomes (PROs) to support culturally appropriate cancer care. The outcomes of this study will be used to better meet the needs of immigrant populations, including the tailoring of interventions appropriate to different health literacy levels. Outcomes will also inform strategies for PR0 assessment to inform unmet needs and to address Australian healthcare system challenges to meet the needs of immigrant populations.

Ethics and dissemination The study was reviewed and approved by the Human Research Ethics Committee of South Western Sydney Local Health District (approval number: HREC/16/LP00L/650). Results from the study will aim to be published at international conferences and in peer-reviewed journals.

\section{Strengths and Limitations}

- Few studies exploring low levels of health literacy in immigrant cancer populations.

- Levels of health literacy evaluated using a validated self-report questionnaire, but other secondary outcome measures not cross-culturally validated.

- 150 English, Arabic and Vietnamese patients with cancer and survivors will be sampled.

- Results will be used to tailor interventions to different health literacy levels.

- Results to inform patient-reported outcome assessment of immigrant patients' unmet needs/ challenges.

\section{INTRODUCTION}

Australia has the third largest immigrant population in the world. ${ }^{1}$ Approximately $28 \%$ of Australian residents were born overseas, with half born in non-English-speaking countries. ${ }^{12}$ Approximately $19 \%$ of the population speak a language other than English at home, with Arabic and Vietnamese among the five most frequently spoken languages. ${ }^{2}$ This poses significant challenges to health services to ensure the delivery of acceptable, equitable and accessible healthcare. ${ }^{23}$

Cancer is the leading cause of disease-related burden in Australia, with approximately a quarter of cancers diagnosed among immigrants. ${ }^{4}$ In 2014, it was projected that 150000 Australians would be diagnosed with cancer each year by $2020 .{ }^{3}$ By international standards, cancer survival rates in Australia are high at $68 \% .{ }^{5}$ However, despite improvements in survival rates, ${ }^{3}$ cancer is still a life-threatening illness that confronts patients with a wide range of complex physical, psychosocial and practical healthcare challenges. ${ }^{6}$ 
Patients have little time to process the news of a diagnosis, including their emotional reactions, before they need to navigate a complex healthcare system, process new information and make difficult treatment decisions. Approximately a third of the patients with cancer experience high levels of physical or psychological distress. ${ }^{7}$ Australian immigrant patients with cancer and caregivers have greater anxiety and depression, and poorer overall quality of life, than their Englishspeaking counterparts. ${ }^{8}$ Immigrant patients with cancer also experience inferior psychosocial outcomes, ${ }^{9}$ higher incidence and less relief of side effects ${ }^{10}$ which is amplified by unfamiliarity with the Australian healthcare system, including being faced with a lack of culturally and linguistically appropriate information, ${ }^{11}$ and difficulties communicating with the treating team, even with the help of interpreters. ${ }^{11} 12$

Potential barriers to optimal cancer care among immigrants include lack of English proficiency, poor health literacy and lack of understanding of medical terminology and of the Australian health system. ${ }^{13}$

Currently, there is limited research on the health literacy of immigrant patients with cancer which may be an important factor in patients feeling misunderstood, isolated and overwhelmed. ${ }^{14}$ Health literacy is defined by the WHO as the "cognitive and social skills which determine the motivation and ability of an individual to gain access to, use and understand information in ways which promote and maintain good health'. ${ }^{15} 16$ Health literacy encompasses more than simply being able to read and understand information, it also includes the ability to access health information and capacity to use it effectively. It is a significant issue for Australia, as health information and systems are becoming increasingly complex ${ }^{6}$ and our immigrant population grows.

Health literacy is a critical factor in empowering patients to manage their own well-being ${ }^{17}$ and improving health outcomes, ${ }^{18}$ particularly in the context of an increasingly complex health system. ${ }^{19}$ However, it is a currently overlooked issue throughout the cancer-care continuum, as the ability to manage health and health literacy is often studied separately from one another. ${ }^{20}$ This may potentially leave patients with low health literacy unable to make personal connections and shape their own health decisions, ${ }^{21}$ resulting in feeling distressed and misunderstood $^{22}$ as they try to interact with their healthcare providers, particularly if such interaction is in a language other than their own. ${ }^{23}$ While this research informs us about the consequences of poorer health literacy in English-speaking patients, we know little about health literacy in immigrant patients.

The aim of this study is to investigate levels of health literacy and correlates of poor health literacy in immigrant populations affected by cancer, relative to their English-speaking counterparts. The specific objectives include:

a. Quantify the levels of health literacy and eHealth literacy in two immigrant populations-Arabic and
Vietnamese - affected by cancer, and their Englishspeaking counterparts.

b. Identify sociodemographic and clinical characteristics associated with poorer health and eHealth literacy.

c. Inform future research aimed at:

i. Using appropriate methods to collect patient-reported outcomes from immigrant populations.

ii. Tailoring existing healthcare information and (self-management) resources to current health literacy levels in immigrant populations.

iii. Developing interventions to improve health literacy levels in immigrant populations.

\section{METHODS/DESIGN}

\section{Study design and setting}

This cross-sectional study will employ a patient self-report questionnaire to explore health literacy levels and factors underlying poor health literacy among Arabic, Vietnamese and English-speaking patients diagnosed with cancer. Study recruitment will be undertaken in South Western Sydney Local Health District (SWSLHD), a culturally diverse district, with $48 \%$ of residents speaking a language other than English; Arabic and Vietnamese being in the top three. ${ }^{24}$

\section{Participants}

Patients will be eligible to participate if they: (a) are aged 18 years or over; (b) have received a cancer diagnosis within the last 5 years; (c) identify English, Arabic or Vietnamese as their primary language spoken; (d) were born outside of Australia (for the Arabic-speaking and Vietnamese-speaking groups) or born in a predominantly Englishspeaking country (eg, Australia, UK, New Zealand, USA) for the English-speaking sample and (e) are cognitively able to provide informed consent. Patients recruited from cancer centres (see the Recruitment section) will be prescreened by their treating oncologist against the study eligibility criteria and clinical judgement.

\section{Recruitment}

Study recruitment will occur via one of two approaches Cancer care centres

Clinic lists of patients who appear to meet eligibility criteria (b) and (d) (see the Participants section) and are receiving care at one of the SWSLHD cancer centres will be generated monthly, sourced from the oncology information system (OIS) and sent to the study team. The lists will be reviewed by attending oncologists to identify eligible patients based on study inclusion criteria and their clinical judgement. Eligible patients will be invited to participate either in clinic or through a mailout approach.

\section{In clinic}

Patients attending an appointment at the cancer care clinic will be invited to participate by a bilingual member of the research team in the waiting area. Research staff will explain the study, confirm eligibility and provide 
patients with a study pack containing all relevant documentation (invitation letter, participant information and consent form, study questionnaire, reply-paid envelope).

\section{Mailout}

Patients identified as eligible by their attending oncologist will be phoned by a bilingual research team member who will introduce the study to the patient and ask whether they would like to receive a study pack containing additional information and materials (see In Clinic section). Patients who agree to receive a pack and report having sufficient written literacy or access to someone to help complete the questionnaire will be sent a pack in their preferred language.

Bilingual research staff will call invitees 2 weeks after study packs are sent to answer questions and prompt invitees to return their consent form and questionnaire if they wish to participate. Five attempts at various times of the day will be made to contact the patient, with patients who cannot be reached after this time marked as 'non-responders'. All patients will require some level of successful contact with the research team before they are marked as non-responders.

\section{Community organisations}

Organisations which support English, Arabic and/or Vietnamese patients with cancer in some capacity will be identified using internet searches and multidisciplinary contacts within the local health district. The research team will approach these organisations for their assistance in identifying and reaching eligible participants; and the principal investigator (MG) will meet with those who express an interest in assisting the project to tailor potential recruitment to suit the organisation's needs. This may include but is not limited to: placing project flyers throughout the organisation, linking interested participants back to the research team for recruitment, preparing study packs for distribution by the organisation to their members or presenting the project at organisation meetings.

\section{Patient and public involvement}

As part of the study design development, community involvement was sought from both English and Arabic cancer research consumer panels representing the South West Sydney region, to explore cultural acceptability, appropriateness and need to address this gap in knowledge. The study design and details were well received, and we will continue to engage with the community to further develop and ensure cultural acceptability and appropriateness of this work.

\section{Measures}

The study questionnaire will be available in English, Arabic and Vietnamese, and will include measures assessing health literacy, healthcare utilisation, self-efficacy and perceived acculturation into the Australian community. The questionnaire will also include items exploring engagement with health services, preferences for information provision, and demographic and clinical information. The Health Literacy Questionnaire (HLQ) ${ }^{16}$ is currently commercially available for use in English, Arabic and Vietnamese. All other measures outlined below are originally validated in English and were translated into Arabic and Vietnamese for use in this study. We are not aware of any cross-cultural validation studies using these measures, and we acknowledge that this is a limitation of the study.

\section{Primary outcome measure}

\section{Health literacy and eHealth literacy}

The primary outcomes of this project will be assessed using the $\mathrm{HLQ}^{16}$ and the eHealth Literacy Scale (eHEALS). ${ }^{25}$ The HLQ focuses on a range of health literacy capabilities, including an individual's experiences and engagement with the healthcare system. The measure was originally informed by the WHO. ${ }^{26}$ eHEALS is designed to and will be used to measure an individual's perceived skills at using the internet. ${ }^{25}$

\section{Health literacy}

The HLQ is a commercially available measure which is valid and reliable ${ }^{162728}$ and available in English, Arabic and Vietnamese. The 44-item tool includes nine domains: feeling understood and supported by healthcare providers, having sufficient information to manage my health, actively managing my health, social support for health, appraisal of health information, ability to actively engage with healthcare providers, navigating the healthcare system, ability to find good health information and understanding health information well enough to know what to do. ${ }^{16}$ HLQ items are rated on two sets of Likert scales, a 4-point scale asking respondents to rate their agreement with statements such as 'I feel I have good information about health' from 'Strongly disagree' (1) to 'Strongly Agree' (4), and a 5-point Likert scale asking respondents to indicate their degree of difficulty in completing tasks such as 'Get health information in words you understand' from 'Cannot do or always difficult' (1) to 'Always easy' (5). ${ }^{16}$ The HLQ generates a score for each domain with higher scores indicating better health literacy. Scores can also be dichotomised into low and high to create a health literacy 'profile' for each patient. ${ }^{16}$

\section{eHealth literacy}

Participants' eHealth literacy levels (ie, knowledge, comfort and perceived skills at finding, evaluating and applying electronic health information to health problems $)^{25}$ will be assessed using the eHEALS. ${ }^{25}$ The eHEALS is a reliable and valid scale ${ }^{25} 29$ that asks about the usefulness of the internet in helping individuals make decisions about health and the importance of being able to access health information online. The eHEALS comprises eight items, such as 'I know how to find helpful health resources on the Internet' that are rated on a 5-point Likert scale ranging from 'strongly agree' (1) to 'strongly 
disagree' (5). ${ }^{25}$ Higher scores indicate greater eHealth literacy.

\section{Correlates of health literacy}

\section{Sociodemographic and clinical characteristics}

Sociodemographic data to be collected include: date and country of birth, year of migration to Australia (for immigrant cohort), postcode, language spoken at home, relationship status, education level and employment status. Clinical characteristics include: years since diagnosis, tumour site, treatment undertaken and information about the patients' comorbidities (ie, high blood pressure, diabetes, heart attack). Some of the clinical information (diagnosis date and site of tumour) will be collected from the OIS, for patients receiving care at participating SWSLHD cancer centres.

During recruitment, the need for an interpreter will be routinely pulled from the OIS, for patients receiving care at participating SWSLHD cancer centres.

Self-efficacy will be measured using the 12-item Communications and Attitudinal Self-Efficacy scale for cancer (CASE-Cancer).$^{30}$ The CASE-Cancer is a reliable, validated ${ }^{30}$ measure of self-efficacy that yields scores on three factors-understanding and participating in care, maintaining positive attitudes, and seeking and obtaining information. The scale has high construct validity and internal consistency. ${ }^{30} 31$ Individuals are asked to indicate their level of agreement at the present time with each of 12 statements, such as 'I know that I will be able to actively participate in decisions about my treatment' and 'I am confident that I am able to deal with any unexpected health problems' using a 4-point Likert scale ('strongly disagree' (1) to 'strongly agree (4)), with higher scores indicating higher self-efficacy in each factor. ${ }^{30}$

Healthcare utilisation will be measured using the 4-item Stanford Health care Utilisation scale. ${ }^{32} 33$ The scale asks individuals questions regarding frequency of visiting their healthcare physician and hospital emergency room and how long they have spent in hospital (nights) over the past 6 months. All items ask for a numerical response and the four questions yield four independent scores. ${ }^{33}$

Acculturation will be measured using the 8-item acculturation scale ${ }^{34}$ which yields a single score. The scale is valid and reliable, and is shown to have high levels of internal consistency and is originally tested in an Arabicspeaking cancer population in Australia. ${ }^{34}$ The scale asks respondents to rate their preferences for language spoken at home and reading and writing (native language vs English), identification within a group (another ethnic group vs Australian only) and how important it is that traditions are honoured or followed ('very important' to 'not at all important'). Scores can be grouped into low, medium and high levels of acculturation with a higher score indicating a higher level of acculturation into Australia society. ${ }^{1134}$
Engagement with health services

Questions regarding the number of doctors regularly seen, private health insurance cover (if applicable), government concession cards held and preferences regarding the type, amount and format of information received (eg, paper, websites, podcasts, verbal information from treatment team) will be included.

\section{Sample size}

A sample size of $n=150$ (50 in each language group (English, Arabic and Vietnamese)) will provide sufficient power $(0.80)$ to determine a $24 \%$ difference in health literacy between English-speaking and non-English-speaking patients, with a significance level of 0.05 and assuming English-speaking patients meet Australian health data means which suggests $44 \%$ of English-speaking Australians have adequate levels of health literacy. ${ }^{35}$

\section{Data analysis}

Descriptive statistics will be used to summarise participant sociodemographic and clinical characteristics and healthcare utilisation. The distribution of total and subscale scores for heath literacy, acculturation and self-efficacy will be described using means and medians with SDs and IQRs.

Multivariable logistic regression will be used to determine differences in immigrant and English-speaking health literacy level as dichotomised into high and low health literacy, in line with the HLQ validation paper. ${ }^{16}$ Analyses will explore associations with age, gender, education level, health insurance status, number of chronic illnesses, language spoken at home and acculturation level which indirectly assesses English proficiency. While exploring the differences in health literacy between language groups, English-speaking ability as represented by need for an interpreter and preferred language will be controlled for.

\section{Ethics and dissemination}

This study adheres to the National Health and Medical Research Council National Statement on Ethical Conduct in Human Research ${ }^{36}$ and the principles of the Declaration of Helsinki. ${ }^{37}$

The study involves vulnerable groups within the community and, so it is imperative that the study is conducted in a sensitive and culturally appropriate manner. On invitation to the study, patients will be given the opportunity to review all study materials in their preferred language and ask any questions about the study. Furthermore, patients who feel overwhelmed or anxious at any point during study participation will be referred to an appropriate support service (eg, Cancer Council Helpline). Patients will also be reassured that they are free to withdraw from the study at any time without reason or consequence.

Results from the study will be disseminated through presentation at international conferences and publications in peer-reviewed journals. 


\section{DISCUSSION AND CONCLUSIONS}

Poor health literacy has been associated with higher use of health services, ${ }^{38}{ }^{39}$ low levels of healthcare knowledge $^{40} 41$ and poorer health outcomes ${ }^{18}$ in various (primarily English-speaking) populations. Specifically, patients with cancer with low health literacy are more likely to have difficulties understanding their disease and diagnosis $^{4142}$ and have poor communication with health professionals which could lead to substandard care. ${ }^{43}$ However, little is known about the levels and correlates of health literacy and eHealth literacy in immigrant cancer populations. Health literacy is a key factor to consider in patient care as it affects one's capacity to make decisions and support the self-management of health.

This study will help inform the gap in knowledge regarding levels of health literacy and correlates of poor health literacy for two of the largest immigrant populations in Australia, Arabic and Vietnamese, compared with their English-speaking counterparts. Specifically, this study may help address the challenges of having poor health literacy and high unmet needs, inform the tailoring of existing healthcare information and self-management resources to current health literacy levels of immigrants and development of interventions targeted at improving the health literacy particularly of immigrants.

The use of standardised measures in the questionnaire will allow comparison of patient health and eHealth literacy with other populations in an aim to gauge the relative needs of immigrant populations affected by cancer and guide delivery of services tailored to immigrant health literacy levels and development of interventions to improve health literacy. Identifying the differences between these groups will allow for the exploration of unmet needs of the immigrant Arabic and Vietnamese communities, and give insight into methods to fulfil these needs.

Acknowledgements The authors thank the Liverpool Cancer Therapy Centre, the Bankstown Cancer Care Centre and all our community organisations for agreeing to facilitate recruitment. Martha Gerges, Ben Smith, Ivana Durcinoska and Afaf Girgis are supported by Cancer Institute NSW grants. CONCERT is funded by Cancer Institute NSW with contributions from University of New South Wales, Western Sydney University, University of Wollongong, Illawarra and Shoalhaven Local Health Districts and the Ingham Institute for Applied Medical Research.

Contributors All authors contributed to study conception and design and have contributed to the preparation and review of the manuscript. MG contributed to the preparation of documents for ethical approval including study materials (questionnaire, participant information statement, etc) to support data collection and managed the liaison with recruitment partners and managed data collection. $A G, A B S$ and ID contributed to study material preparation and review prior to ethical approval. AG has ownership over the funds allocated to this study, as director of the Psycho-Oncology Research Group at the Centre for Oncology Education and Research Translation (CONCERT), Ingham Institute for Applied Medical Research, The University of New South Wales, Australia. HY contributed to data collection and managed the liaison with recruitment partners and managed data collection.

Funding This study was funded internally from the Psycho-Oncology Research Group, Centre for Oncology Education and Research Translation (CONCERT), Ingham Institute for Applied Medical Research, University of New South Wales, Australia.

Competing interests None declared.

Patient consent Not required.
Ethics approval The study was reviewed and approved by the Human Research Ethics Committee of South Western Sydney Local Health District, Sydney Australia (approval number: HREC/16/LP00L/650) and by the Higher Degree Research Committee at the University of New South Wales, Australia.

Provenance and peer review Not commissioned; externally peer reviewed.

Open access This is an open access article distributed in accordance with the Creative Commons Attribution Non Commercial (CC BY-NC 4.0) license, which permits others to distribute, remix, adapt, build upon this work non-commercially, and license their derivative works on different terms, provided the original work is properly cited, appropriate credit is given, any changes made indicated, and the use is non-commercial. See: http://creativecommons.org/licenses/by-nc/4.0/.

\section{REFERENCES}

1. OECD. Foreign-born population. 2017 http://dx.doi.org/10.1787/ 5a368e1b-en

2. Australian Bureau of Statistics. Cultural diversity in Australia. Cultural diversity in Australia. Reflecting a nation: stories from the 2011 Census. 2012. http://www.abs.gov.au

3. Australian Institute of Health and Welfare \& Australasian Association of Cancer Registries. Cancer in Australia: an overview 2012. Canberra: Australian Institute of Health and Welfare, 2012. Cancer series no. 74. Cat. no. CAN 70.

4. Supramaniam R, O'Connell DL, Tracey EA, et al. Cancer incidence in New South Wales migrants 1991 to 2001. Sydney: Cancer Council NSW, 2006.

5. Australian Institute of Health and Welfare. AloHa W, ed. Australian Cancer Incidence and Mortality (ACIM) books: All cancers combined. Canberra, 2017.

6. Lambert SD, Harrison JD, Smith E, et al. The unmet needs of partners and caregivers of adults diagnosed with cancer: a systematic review. BMJ Support Palliat Care 2012;2:224-30.

7. Dunn J, Ng SK, Holland J, et al. Trajectories of psychological distress after colorectal cancer. Psychooncology 2013;22.

8. Butow PN, Aldridge L, Bell ML, et al. Inferior health-related quality of life and psychological well-being in immigrant cancer survivors: a population-based study. Eur J Cancer 2013;49:1948-56.

9. Luckett T, Goldstein D, Butow PN, et al. Psychological morbidity and quality of life of ethnic minority patients with cancer: a systematic review and meta-analysis. Lancet Oncol 2011;12:1240-8.

10. Yi JK, Swartz MD, Reyes-Gibby CC. English proficiency, symptoms, and quality of life in Vietnamese- and Chinese-American breast cancer survivors. J Pain Symptom Manage 2011;42:83-92.

11. Butow PN, Sze M, Dugal-Beri $P$, et al. From inside the bubble: migrants' perceptions of communication with the cancer team. Support Care Cancer 2010;19:281-90.

12. Butow PN, Goldstein D, Bell ML, et al. Interpretation in consultations with immigrant patients with cancer: how accurate is it? J Clin Oncol 2011;29:2801-7.

13. Chou FY, Kuang LY, Lee J, et al. Challenges in Cancer Selfmanagement of Patients with Limited English Proficiency. Asia Pac J Oncol Nurs 2016;3:259-65.

14. Ashing-Giwa KT, Padilla G, Tejero J, et al. Understanding the breast cancer experience of women: a qualitative study of African American, Asian American, Latina and Caucasian cancer survivors. Psychooncology 2004;13:408-28.

15. Organization WH. Health Promotion Track 2: Health literacy and health behaviour, 2009.

16. Osborne RH, Batterham RW, Elsworth GR, et al. The grounded psychometric development and initial validation of the Health Literacy Questionnaire (HLQ). BMC Public Health 2013;13:658.

17. Nutbeam D. Health literacy as a public health goal: a challenge for contemporary health education and communication strategies into the 21st century. Health Promot Int 2000;15:259-67.

18. Omachi TA, Sarkar U, Yelin EH, et al. Lower health literacy is associated with poorer health status and outcomes in chronic obstructive pulmonary disease. J Gen Intern Med 2013;28:74-81.

19. Koay K, Schofield P, Jefford M. Importance of health literacy in oncology. Asia Pac J Clin Oncol 2012;8:14-23.

20. Sentell T, Braun KL, literacy L. limited english proficiency, and health status in asians, latinos, and other racial/ethnic groups in california, 2012:82-99.

21. Nutbeam D. The evolving concept of health literacy. Soc Sci Med 2008;67:2072-8.

22. Jefford M, Tattersall MHN. Informing and involving cancer patients in their own care. Lancet Oncol 2002;3:629-37. 
23. Taira DA. Improving the health and health care of non-englishspeaking patients. J Gen Intern Med 1999;14:322-3.

24. South Western Sydney Local Health District. Health profile of local communities: South Western Sydney Local Health District, 2014.

25. Norman CD, Skinner HA. eHEALS: The eHealth Literacy Scale. $J$ Med Internet Res 2006;8:e27.

26. Nutbeam D. Health Promotion Glossary. Health Promot Int 1998;13:349-64.

27. Maindal HT, Kayser L, Norgaard O, et al. Cultural adaptation and validation of the Health Literacy Questionnaire (HLQ): robust ninedimension Danish language confirmatory factor model. Springerplus 2016;5:1-16.

28. Kayser L, Hansen-Nord NS, Osborne RH, et al. Responses and relationship dynamics of men and their spouses during active surveillance for prostate cancer: health literacy as an inquiry framework. BMC Public Health 2015;15:741.

29. Nguyen J, Moorhouse M, Curbow B, et al. Construct Validity of the eHealth Literacy Scale (eHEALS) Among Two Adult Populations: A Rasch Analysis. JMIR Public Health Surveill 2016;2:e24.

30. Wolf MS, Chang C-H, Davis T, et al. Development and validation of the Communication and Attitudinal Self-Efficacy scale for cancer (CASE-cancer). Patient Educ Couns 2005;57:333-41.

31. Jean-Pierre P, Fiscella K, Freund KM, et al. Structural and reliability analysis of a patient satisfaction with cancer-related care measure: A multisite patient navigation research program study. Cancer 2011;117:854-61.

32. Ritter PL, Stewart AL, Kaymaz H, et al. Self-reports of health care utilization compared to provider records. J Clin Epidemiol $2001 ; 54: 136-41$.
33. Center S-MR. Health Care Utilization Scale Palo Alto. California: Stanford University, 2017. https://www.selfmanagementresource. com/docs/pdfs/English_-_health_care_utilization.pdf2017.

34. Rissel C. The development and application of a scale of acculturation. Aust N Z J Public Health 1997;21:606-13.

35. Statistics ABo. Australian Social Trends, June 2009: Health Literacy Canberra. Australia, 2009. http://www.abs.gov.au/AUSSTATS/abs@. nsf/Lookup/4102.0Main+Features20June+2009.

36. The National Health and Medical Research Council the Australian Research Council and the Australian Vice-Chancellors' Committee. National Statement on Ethical Conduct in Human Research. Canberra: Australian Government, 2007. Updated March 2014. nhmrc.publications@nhmrc.gov.au. (accessed 20 June 2015).

37. Goodyear MDE, Krleza-Jeric K, Lemmens T. The Declaration of Helsinki. BMJ 2007;335:624-5.

38. Schulz PJ, Fitzpatrick MA, Hess A, et al. Effects of eHealth Literacy on General Practitioner Consultations: A Mediation Analysis. J Med Internet Res 2017;19:e166.

39. Berkman ND, Sheridan SL, Donahue KE, et al. Low Health Literacy and Health Outcomes: An Updated Systematic Review. Ann Intern Med 2011;155:97.

40. Schillinger $D$, et al. Association of Health Literacy With Diabetes Outcomes. JAMA 2002;288:475-82.

41. Williams MV, Baker DW, Parker RM, et al. Relationship of functional health literacy to patients' knowledge of their chronic disease. A study of patients with hypertension and diabetes. Archives of internal medicine 1998;158:166.

42. Gazmararian JA, Williams MV, Peel J, et al. Health literacy and knowledge of chronic disease. Patient Educ Couns 2003;51:267-75.

43. McCray AT. Promoting health literacy. J Am Med Inform Assoc 2005;12:152-63. 